\title{
A primer on the relationship between group size and group performance
}

Mauricio Cantor ${ }^{1,2,3,4^{*}}$, Lucy M. Aplin $3,5,6+$, Damien R. Farine ${ }^{2,3,7+^{*}}$

${ }^{1}$ Department for the Ecology of Animal Societies, Max Planck Institute of Animal Behavior, Konstanz, Germany

2 Department of Biology, University of Konstanz, Germany

${ }^{3}$ Centre for the Advanced Study of Collective Behaviour, University of Konstanz, Germany

${ }^{4}$ Departamento de Ecologia e Zoologia, Universidade Federal de Santa Catarina,

Brazil

${ }^{5}$ Cognitive and Cultural Ecology, Max Planck Institute of Animal Behavior, Konstanz, Germany

${ }^{6}$ Edward Grey Institute of Field Ornithology, Department of Zoology, University of Oxford, UK

${ }^{7}$ Department of Collective Behaviour, Max Planck Institute of Animal Behavior, Konstanz, Germany

† These authors contributed equally

*Corresponding authors: mcantor@ab.mpg.de,dfarine@ab.mpg.de

Max Planck Institute of Animal Behavior, Am Obstberg 1, Radolfzell 78315, Baden-Württemberg, Germany 


\section{ABSTRACT}

Living in groups can benefit individuals in many ways, including in innovative problem solving. Several hypotheses have suggested mechanisms to explain why larger groups disproportionately outperform smaller groups, including the skill pool and pool of competence effects. However, disentangling these potential mechanisms from the effects of group size alone has been challenging. Here, we first outline key ways in which group size can shape performance in innovative problem-solving. We then detail the non-linear nature of the mathematical relationship between group size and various measures of group performance. Finally, we use simulations to confirm that measures of group performance in innovative problem-solving scale non-linearly with group size, even in the absence of any other effect. Our study provides guidance on how best to evaluate hypotheses about group composition on innovative problem-solving, and clarity to help future studies make appropriate assumptions when developing null hypotheses against which to test their empirical data.

Key words: innovation, learning, pool of competence, problem solving, skill pool, social learning, swarm intelligence

Many animals live in dynamic environments where they are constantly faced with new challenges. Behavioural innovations are key short-term responses to challenges, with novel or modified behavioural variants allowing individuals to fine-tune their behavioural repertoires to new local conditions and resource distributions (Sol et al., 2002; Reader and Laland, 2003; Griffin and Guez, 2014). For instance, a foraging innovation-whereby an individual adds novel food 
items to its diet or exploits a known item in a novel way-can facilitate invasion or persistence of innovators in novel environments (Sol et al., 2005; Ducatez et al., 2020). Behavioural innovations can also be associated with social behaviour (see Reader and Laland, 2003). For one, by living in groups, individuals benefit from the well-known effects such as the dilution of risk and the social transfer of information and knowledge (e.g. Caraco, 1981). As such, innovativeness may also be related to group size. Group-living can reduce the ability to learn—or the benefit of learning-due to competition or scrounging (Hirsch, 2007). Conversely, group-living can promote superior innovative problem-solving, with individuals in larger groups solving novel problems and producing innovations disproportionately faster and/or more often than smaller groups (Pitcher et al., 1982; Couzin et al., 2005; Berdahl et al., 2013).

The relationship between group size and extraction of resources from the environment is well explored from an individual-centric approach, for example asking 'how much energy does an individual in a group size of $n$ accumulate over time?' (Caraco, 1981; Clark and Mangel, 1986; Ranta et al., 1993). More recently, the interest has shifted to testing how foraging groups deal with new events'has any individual innovated to access a new resource?'. Both field and experimental studies now suggest that larger groups are more efficient at innovative problem-solving when confronted with new food sources (Kerr and Tindale, 2004; Liker and Bokony, 2009; Morand-Ferron and Quinn, 2011; Ashton et al., 2019). For example, in Liker and Bokony (2009) groups of 6 captive house sparrows (Passer domesticus) were on average 11 times faster to solve a foraging task than groups of 2, and all members of larger groups received food 7 
times faster. Similarly, Ashton et al. (2019) found a non-linear correlation between the time to first innovation and groups size in wild Australian magpies (Cracticus tibicen), with solutions emerging faster in larger groups (group sizes of 2 to 11). It should be noted that being among many conspecifics can sometimes slow down or limit opportunities for individuals to engage in innovative behaviour (Overington et al., 2009; Griffin et al., 2013). Thus, counterexamples exist to highlight that the direction of the relationship between group size and performance is not always clear (e.g. Stöwe et al., 2006; Thornton and Samson, 2012; Thornton and Malapert, 2009).

While empirical studies have yielded important insights into the individual benefits of living in a larger group, our understanding of these studies has been hampered by the lack of clarity on the underlying mechanisms driving the observed outcomes, and on the statistical patterns that different mechanisms are expected to produce. Our aim here is to provide clarification on the fundamentals of the relationship between group size and measures of group performance on innovative problem-solving, whereby performance is measured by latency or propensity to innovate. First, we discuss the multiple hypotheses that have arisen to explain the relationship between group size and rates or propensity for innovations. Second, we highlight that the basic mathematical relationship between group size and group performance can sometimes be linear and sometimes be non-linear, depending on the type of actions that are being measured. Finally, we use a simple model to explore how the non-linear relationships between group size and the time taken for innovations to emerge and to spread within social groups affects our ability to test some of the 
mechanisms hypothesised to underlie group-size effects on the propensity to innovate. Our study highlights that non-linear functions (i) are expected rather than surprising in studies of group performance, and (ii) make it difficult to quantify the role of alternative hypotheses. Our results suggest that variation in behavioural composition is likely to play a relatively minor role in the relationship between groups size and innovation in all except the smallest groups. Rather, the mechanisms underlying the benefits of larger group sizes for innovation are largely simple and direct, with most circumstances not requiring invoking complex hypotheses.

\section{Why do larger groups outperform smaller groups?}

In theory, many drivers can lead to superior innovative problem-solving performance in large groups (Table 1). First, larger groups can solve a problem faster or more often because there is a clear effect of the additional effort of multiple individuals on the solving probability. This phenomenon is often referred to as 'swarm intelligence', or 'collective cognition' (e.g. Krause et al., 2010). Second, individuals can directly cooperate to solve a problem-and being in larger numbers could facilitate recruiting good collaborators (see also Melis et al., 2006). Third, there may be an indirect effect of the social context; for example, individuals in a larger group may have less aversion to novelty and be at lower risk of predation. Lower neophobia or reduced predator vigilance can then increase the chance for-or the time available to invested in-behaviours that lead to innovations (Ryer and Olla, 1992; Coleman and Mellgren, 1994; Stöwe et al., 2006; Morand-Ferron and Quinn, 2011). Being surrounded by many 
conspecifics can increase cognitive performance (Thornton and Samson, 2012;

Ashton et al., 2018; see also the 'social intelligence hypothesis' e.g. Reader and Laland, 2002); and it can also increase motivation to solve a problem (social facilitation) due to exposure to attempts and subsequent competition (Soma and Hasegawa, 2004).

One prominent hypothesis to explain increased performance in larger groups proposes that the probability of innovation is affected by emergent or preexisting group heterogeneity, known respectively as the 'skill pool' and 'pool of competence' effects. The skill pool effect implies that competition in larger groups will lead individuals to specialise on distinct foraging behaviours, representing a larger collective repertoire and translating into improved search efficiency and joint discoveries of food (Giraldeau, 1984). The pool of competence effect, instead, implies that larger groups are more likely to be composed of phenotypically diverse members (Liker and Bokony, 2009; Morand-Ferron and Quinn, 2011) and-given that group diversity can leverage the chance of success (Hong and Page, 2004) — by chance, larger groups are more likely to contain individuals with the skills to solve a given problem efficiently. In turn, members of larger groups will be more likely to benefit from the skill of these members of their group. Traits that are known to influence innovation include age, sex, dominance rank, motor tendencies, cognitive ability, and personalities (Burns and Dyer, 2008; Biondi et al., 2010; Morand-Ferron and Quinn, 2011; Thornton and Samson, 2012; Griffin and Guez, 2014; Keynan et al., 2015; Amici et al., 2019; Gibelli et al., 2019; Smit and van Oers, 2019). 
Table 1. Common hypotheses for the positive effect of group size on the problem-solving performance and the emergence of innovative behaviour (For references in support or against these hypotheses, please see the text).

\begin{tabular}{ll}
\hline Hypothesis & Effect \\
\hline $\begin{array}{l}\text { Swarm } \\
\text { intelligence }\end{array}$ & $\begin{array}{l}\text { Groups combine and process information independently collected by } \\
\text { individuals, providing collective solutions to a problem which is unavailable } \\
\text { to small groups or individuals alone }\end{array}$ \\
$\begin{array}{l}\text { More } \\
\text { collaboration } \\
\text { opportunities }\end{array}$ & $\begin{array}{l}\text { Problems may require collaboration to be solved and individuals in larger } \\
\text { groups have access to a greater pool of potential collaborators }\end{array}$ \\
$\begin{array}{l}\text { Reduced } \\
\text { neophobia }\end{array}$ & $\begin{array}{l}\text { Individuals in larger groups have less aversion to novelty and so can be more } \\
\text { prone to exploration and to attempt to solve novel problems }\end{array}$ \\
$\begin{array}{l}\text { Reduced } \\
\text { predator } \\
\text { vigilance }\end{array}$ & $\begin{array}{l}\text { Individuals in larger groups experience lower predation risk and can reduce } \\
\text { their predator vigilance, which facilitates investing in innovative problem }\end{array}$ \\
$\begin{array}{l}\text { Increased } \\
\text { sognitive } \\
\text { performance }\end{array}$ & $\begin{array}{l}\text { Living in groups either selects for cognition, or promotes cognitive } \\
\text { development, which is useful for problem solving (see also the 'social } \\
\text { intelligence hypothesis') }\end{array}$ \\
$\begin{array}{l}\text { Social } \\
\text { facilitation }\end{array}$ & $\begin{array}{l}\text { Individuals in larger groups are more motivated to solve a problem due to } \\
\text { exposure to solving attempts, solutions, and/or competition }\end{array}$ \\
$\begin{array}{l}\text { Skill pool effect } \\
\text { Larger groups experience more competition, thus promote behavioural } \\
\text { specialisation, enlarging the collective repertoire and improving joint } \\
\text { discoveries }\end{array}$ \\
$\begin{array}{l}\text { Pool of } \\
\text { competence }\end{array}$ & $\begin{array}{l}\text { Larger groups are composed by diverse individuals in traits that increase } \\
\text { problem-solving ability thus are likely to contain skilful individuals }\end{array}$ \\
\hline
\end{tabular}

The simple mechanism of greater performance by larger groups

The positive effect of group size and diversity on innovative problem-solving has received support from both theoretical and experimental studies. Theoretical work suggests that individuals should vary in behavioural repertoires and competence (Bolnick et al., 2003; Sih et al., 2019), and that group diversity facilitates innovative problem-solving that exceed individual capacities (e.g. Hong and Page, 2004; Krause et al., 2010). A few empirical studies have also provided evidence for an effect of diversity by comparing groups of the same size 
but differing composition. For example, mixed-species passerine flocks, which have high phenotypic diversity, are more likely to approach and use a novel feeder than similar sized flocks of single species (Freeberg et al., 2017). More directly, empirical comparison of bird flocks have consistently shown that larger flocks outperform smaller flocks in foraging tasks by solving them faster and more often (Liker and Bokony, 2009; Morand-Ferron and Quinn, 2011; Ashton et al., 2019). As compelling as the evidence for greater innovative problem-solving performance in larger groups may be, an alternative mechanism is often overlooked.

The improved performance of larger groups can result from a simpler effectthat larger groups contain more individuals that are available to make solving attempts, and that this higher number of potential attempts per unit time can, in and of itself, result in greater group performance. In other words, larger groups should disproportionately outperform smaller groups without requiring any variation among group members. Indeed, a common misinterpretation of various hypotheses about the effects of group size on group performance has been thatall else being equal—there should be a linear relationship between group size and performance, e.g. that a group of 10 individuals should solve a problem, on average, twice as fast as a group of 5 individuals. This then implies that nonlinear relationships indicate the presence of some additional effect related to variation among group members or social facilitation (e.g. reduction in vigilance). 
The error in the assumption of linearity comes from confounding the additive and multiplicative nature of probabilities. In some cases, increasing group size will have additive effects on group performance. For example, when considering the amount of food items taken by a group of foragers, a group of $n$ individuals will take $\left(n \cdot P_{F}\right)$ food items, where $P_{F}$ is the average number of food items that a single individual takes per unit time. Thus, the effect of increasing group size on the amount of food items taken is linear. By contrast, if we consider the probability of any one group member performing a specific action per unit time, then the relationship between group size and performance becomes non-linear.

Take a simple case where all individuals have the same probability of innovating to solve a problem. The probability of individuals independently not solving such a problem in a given unit time is

$$
P(\text { not solve } \mid n)=\left(1-P_{I}\right)^{n}
$$

where $n$ is the number of individuals in the group and $P_{I}$ is the independent individual-level probability of solving per unit time. This makes the probability that any given individual solves the same problem in the same unit time as

$$
P(\text { solve } \mid n)=1-\left(1-P_{I}\right)^{n}
$$

in a single attempt, and

$$
P(\text { solve } \mid n)=1-\left(1-P_{I}\right)^{n \cdot t}
$$

in $t$ attempts (e.g. in $t$ units of time). In other words, the probability that a group solves a problem is based on the innovation rate raised to the power of the effort (group size times the number of attempts), making the relationship between group size and the probability to solve per unit(s) time multiplicative (i.e. nonlinear, Figure 1). Should group members have opportunities to copy an 
innovator, then the probability of an individual solving in a given time step

becomes

$$
1-\left(1-P_{I}\right) \cdot\left(1-P_{L}\right)^{n}
$$

where $P_{L}=1-\left(1-P_{S}\right)^{n_{S}}$, which is the probability of socially learning per group member given $n_{s}$ demonstrators at that time step. Thus, if individuals can then learn from one another, the time taken for all group members to solve the problem will decrease, but the basic shape of this relationship will not change.

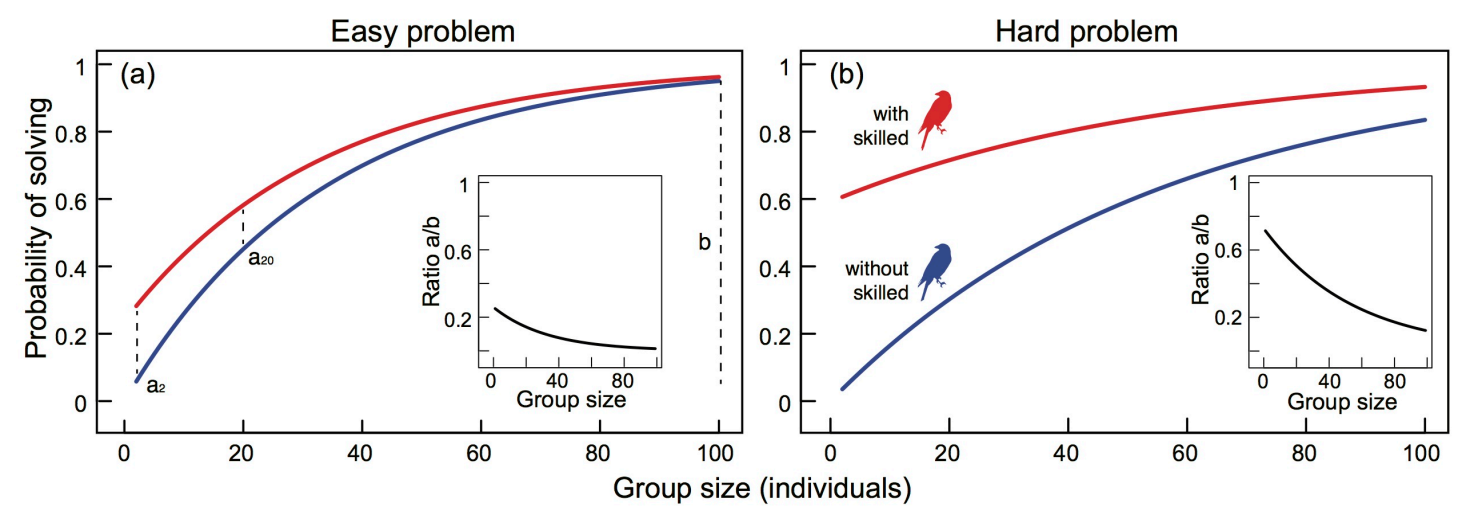

Figure 1: The mathematical relationship between group size and group performance. The relationship is shown for (a) an easy problem where a skilled individual is 10 times more likely to solve per unit effort than an unskilled individual; and for a (b) hard problem where skilled individuals are 50 times more likely than unskilled individuals to solve the problem. The probability of at least one individual solving the easy and the hard problems scales non-linearly with number of individuals in the group both with (red) and without a skilled individual (blue). The influence of a skilled individual in the group performance in solving the problem will therefore be decreasingly important as group size increases, and become insignificant in large groups. Inset plots indicate the relative effects of the presence of a skilled individual in the group versus the effects of increasing group size on the probability of solving a problem (Ratio $\frac{a_{n}}{b}$ ), where $a_{n}$ represents the difference in the probability of solving between groups with (red) and without a skilled individual (blue) when group size is $n$, and $b$ represent the increase in the probability of solving in small (here $n=2$ ) versus large (here $n=100$ ) groups without a skilled individual (blue). 
Now, consider that individuals inherently differ in their past experiences, behavioural repertoires, and/or motor abilities - a requisite for the pool of competence effect (Morand-Ferron and Quinn, 2011). When some individuals are more skilled in innovative problem-solving than others, the probability of solving the same problem after multiple attempts becomes

$$
P(\text { solving } \mid n)=1-\left(1-P_{I}(u)\right)^{n_{u} \cdot t} \cdot\left(1-P_{I}(s)\right)^{n_{s} \cdot t}
$$

where $n_{u}$ is the number of unskilled individuals, $n_{S}$ is the number of skilled individuals, $P_{I}(u)$ is the probability of an unskilled individual solving a problem per unit time, and $P_{I}(s)$ is the probability that a skilled individual solves per unit time. Note that adding skilled individuals would not change the basic nature (i.e. non-linearity) of the group size and performance relationship.

The multiplicative nature of the group size and performance relationship means that while having skilled individuals in a group can make differences in the performance among very small groups, as group size increases, the contributions of skilled individuals becomes disproportionally smaller (groups with or without skilled individuals become indistinguishable, Figure 1a). By contrast, adding more group members can greatly enhance the probability of solving, with this relationship levelling off. At which point group size no longer contributes to an increase in the probability of solving relationship will be impacted by a range of factors, including boundary effects (the minimum time to solve even if a problem is solved immediately), and social effects such as interference among individuals. We do not consider these further as they will not affect the overall non-linear nature of the relationship. 
The non-linear relationship between group size and group performance at solving a problem means that the shape of the relationship is not prescriptive of the pool of competence effect (or other indirect effects). Thus, care is required to ensure that correct assumptions are made when developing the null hypothesis for group behaviours.

\section{Exploring the group size versus group performance relationship}

We demonstrate the expected relationships between group size and two measures of group performance by building a simple model. In our model, animal populations are composed of skilled and unskilled individuals, and individuals form groups of various sizes. We then simulate individuals attempting to solve a problem independently. Once the problem is solved, individuals can continue to solve independently but can also copy others. After all individuals have attempted, we record the group size, the time step, and the number of individuals that have solved the problem by that time step. We evaluated group performance both in terms of 1) the time taken to achieve the solution (solving or innovation efficiency) and 2) by the proportion of the group that was successful over repeated time steps.

In our model, we vary four parameters: group size, presence of a skilled individual, individual probability of independently solving per time unit, and probability of socially learning from the innovator. We simulated 1,000 replicated populations in which individuals formed groups of sizes spanning from 2 to 75 members $(G \in\{2,3,5,7,10,13,16,20,24,28,32,38,44,50,60$, 
75\}) are given a problem to solve until 100,000 time steps. Groups could contain or not a skilled individual. Skilled individuals have a probability of independent solving $\mathrm{P}_{\text {skilled }}($ solving $)=0.01$, and so are expected to solve the problem relatively often. When the solution to the problem is relatively easy, $\mathrm{P}_{\text {skilled }}$ (solving) is 10 -fold higher the probability of independent solving by an unskilled individual, $\mathrm{P}_{\text {unskilled }}$ (solving) $=0.001$; when the problem is hard, $\mathrm{P}_{\text {skilled }}$ (solving) is 50-fold higher than $\mathrm{P}_{\text {unskilled }}$ (solving) $=0.0002$. The probability of any individual, skilled or not, to copy the solution from each other was set as $\mathrm{P}($ learning $)=0.1$. Our simulations produce three clear results: 1$)$ both measures of group performance increase with group size; 2) the effect of individual variation in skill level is secondary to the group size effect, and is contingent on group size; 3 ) the difficulty of the problem extends the time to reach a solution, but it does not change the fundamental relationship between group size and performance.

The first outcome of our simulations demonstrates that solving efficiency decreases non-linearly with group size, and that the shape of these curves fits an exponential decay model (Figure 2a,b). When comparing the fit of models to the latency to solve by groups of different sizes, a likelihood ratio test reveals that models with non-linear terms fit significantly better than models without them (Table 2). For groups without skilled individuals, the most important contributor to the fit is the non-linear term $\left(\frac{x}{\text { group.size }}\right.$, where $x$ is a constant fitted by a nonlinear least-squares estimate)—all models that have this term fit equally well (models 1-3, Table 2) and better than all linear models without it (models 4-6, Table 2). By contrast, when groups contain skilled individuals, the linear term 
( $z *$ group. size, where $z$ is a constant) becomes a significant contributor to the fit (model 7 vs. models 8-12, Table 2), along with the non-linear term. Thus, in neither case do linear models better explain the relationship between group size and solving efficiency. Further, it is only situations where groups perform the task efficiently (with low latency), such as when they contain skilled individuals, that the relationship appears more linear (see red line in Figure $2 b$ that contains a significant linear component).

Table 2. Likelihood ratio test (LRT) of alternative models describing the relationship between group size and the latency to solve a problem.

\begin{tabular}{|c|c|c|c|c|c|c|c|c|c|}
\hline \multirow{2}{*}{\multicolumn{2}{|c|}{$\begin{array}{c}\text { Fitted model } \\
\text { Without skilled individuals }\end{array}$}} & \multicolumn{4}{|c|}{ Easy problem } & \multicolumn{4}{|c|}{ Hard problem } \\
\hline & & \multirow{2}{*}{ Df } & \multirow{2}{*}{$\begin{array}{l}\begin{array}{l}\text { Log- } \\
\text { likelihood }\end{array} \\
-54.366\end{array}$} & \multirow[t]{2}{*}{$X^{2}$} & \multirow[t]{2}{*}{$P$} & \multirow{2}{*}{ Df } & \multirow{2}{*}{$\begin{array}{l}\text { Log- } \\
\text { likelihood } \\
-77.270\end{array}$} & \multirow[t]{2}{*}{$X^{2}$} & \multirow[t]{2}{*}{$P$} \\
\hline 1 & $L \sim \frac{x}{g}+(z \cdot g)+c$ & & & & & & & & \\
\hline 2 & $L \sim \frac{x}{g}+c$ & 3 & -55.456 & 2.179 & 0.140 & 3 & -77.277 & 0.015 & 0.902 \\
\hline 3 & $L \sim \frac{x}{g}$ & 2 & -56.639 & 2.368 & 0.124 & 2 & -77.278 & 0.002 & 0.967 \\
\hline 4 & $L \sim(z \cdot g)+c$ & 3 & -92.277 & 71.275 & $<0.001$ & 3 & -116.241 & 77.925 & $<0.001$ \\
\hline 5 & $L \sim(z \cdot g)$ & 2 & -99.637 & 14.720 & $<0.001$ & 2 & -124.292 & 16.102 & $<0.001$ \\
\hline 6 & $L \sim c$ & 2 & -96.242 & 6.789 & $<0.001$ & 2 & -120.606 & 7.371 & $<0.001$ \\
\hline \multicolumn{2}{|c|}{ With skilled individuals } & Df & $\begin{array}{l}\text { Log- } \\
\text { likelihood }\end{array}$ & $X^{2}$ & $P$ & Df & $\begin{array}{l}\text { Log- } \\
\text { likelihood }\end{array}$ & $X^{2}$ & $P$ \\
\hline 7 & $L \sim \frac{x}{g}+\underset{x}{(z \cdot g)+c}$ & 4 & -40.98 & & & 4 & -43.038 & & \\
\hline 8 & $\begin{array}{c}L \sim \frac{n}{g}+c \\
x\end{array}$ & 3 & -50.599 & 19.238 & $<0.001$ & 3 & -56.644 & 27.212 & $<0.001$ \\
\hline 9 & $L \sim \frac{\sim}{g}$ & 2 & -62.867 & 24.537 & $<0.001$ & 2 & -78.21 & 43.133 & $<0.001$ \\
\hline 10 & $L \sim(z \cdot g)+c$ & 3 & -57.131 & 11.472 & $<0.001$ & 3 & -44.623 & 67.175 & $<0.001$ \\
\hline 11 & $L \sim(z \cdot g)$ & 2 & -76.756 & 39.251 & $<0.001$ & 2 & -81.59 & 73.934 & $<0.001$ \\
\hline 12 & $L \sim c$ & 2 & -67.414 & 18.685 & $<0.001$ & 2 & -63.049 & 37.083 & $<0.001$ \\
\hline
\end{tabular}

The time until one individual in a group solves a problem (latency, $L$ ) as a function of group size $(g)$ was fitted to 6 models containing a non-linear ( $x$ ), a linear ( $z$ ), and/or a constant (c) term. The probability of a skilled individual solving an easy problem was 10-fold that of an unskilled individual, and of solving a hard problem was 50-fold. Statistical significance $(P<0.005)$ indicates rejection of the null hypothesis that a sub model (2-6 and 8-12) provides a better fit than the full models ( 1 and 7 , respectively). $D f$ : degrees of freedom, $\chi^{2}$ : likelihood ratio Chisquared statistic. Data from simulations in Figure 2a,b. 

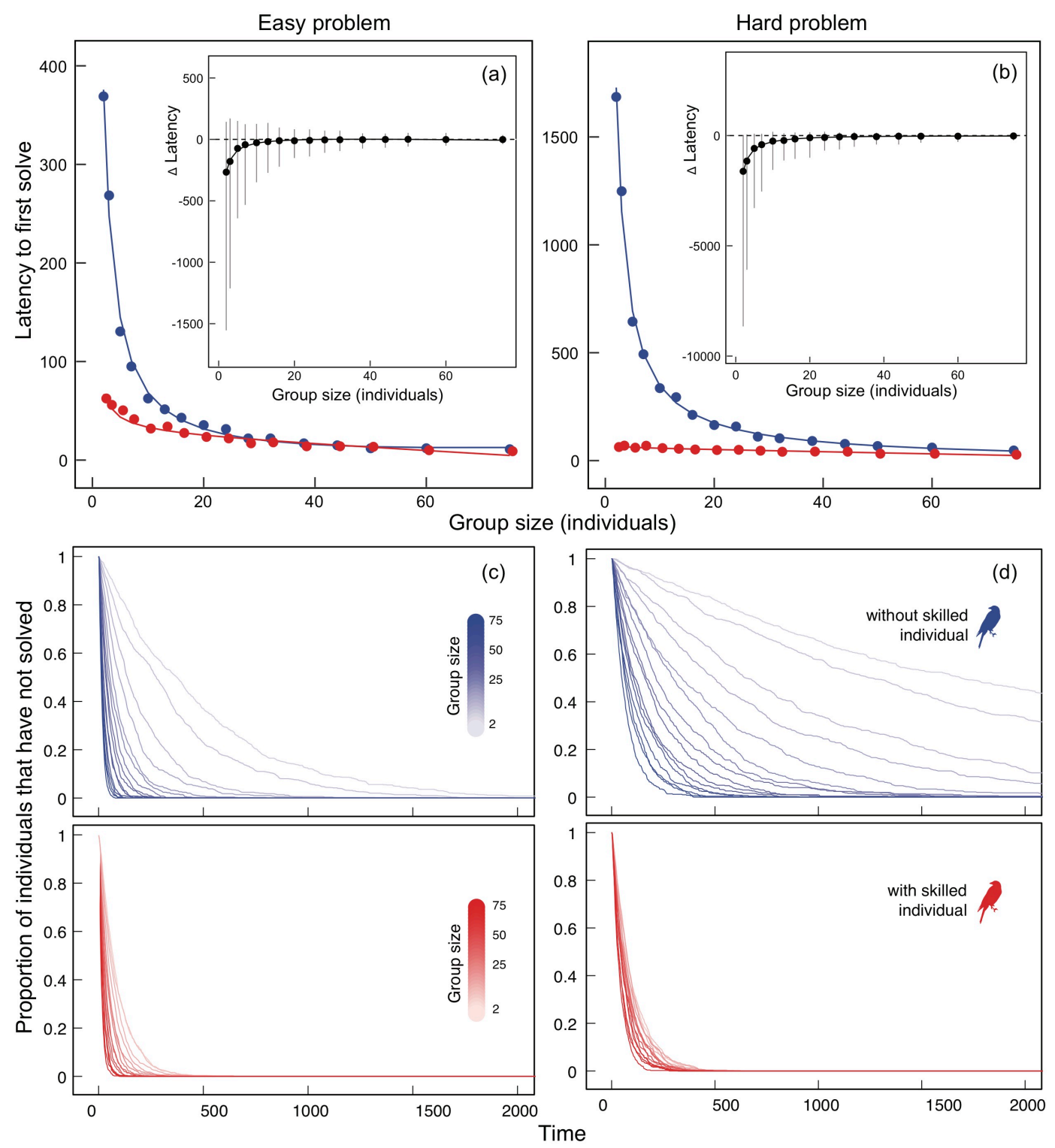

Figure 2: Larger groups perform inherently better, regardless of the members' skill level. Groups containing skilled individuals do not always perform better when solving a given problem than those that do not contain a skilled individual, with the average performance becoming indistinguishable when groups are large. (a) The time until one individual in a group solves a relatively easy problem (y-axis) decreases non-linearly with group size both for groups with (red) and without skilled individuals (blue). For the easy problem, the solving probability of a skilled individual was 10 -fold that of an unskilled individual. Points show the means of 1000 simulated populations with group sizes varying from 2 to 75 individuals; lines show the fit of an exponential decay model (models 1 and 7 from Table 1 for unskilled and skilled groups, respectively). Inset plot shows that the difference in performance ( $\Delta$ latency) by groups with and without skilled individuals is statistically indistinguishable, especially for larger groups, as the confidence intervals (whiskers) always overlap with the horizontal dashed line indicating no 
time difference $(y=0)$. (b) The proportion of individuals in the groups that have not solved the problem decreases with time, and it decreases faster in larger groups, both for groups with (red) and without skilled individuals (blue). Colour shade indicate group size (light=small, dark=large); simulations run for 100,000 times steps but truncated at $\mathrm{t}=2,000$ for better visualization. (c) These patterns are consistent when the problem is hard (skilled individual's solving probability was 50 -fold that of an unskilled), only that (c) it takes longer for any individual to reach a solution, and (d) that members in small groups without skilled individuals takes particularly longer (however, this distinction disappears when groups are very large).

The second outcome of the simulations demonstrates that the time difference in solving between groups with skilled and unskilled individuals is similar across all group sizes, except for very small groups (Figure 2). Further, the number of individuals that have not solved a problem over a given time also decreases with group size; but while this proportion is distinct between small groups with and without a skilled individual, and becomes indistinguishable when the group sizes are large (Figure 2c,d). The third outcome demonstrates that while the members of larger groups solve the problem sooner than those in smaller groups, the presence of skilled individuals in a group only makes a difference when the problem to solve is considerably harder, and so skilled individuals have a much higher probability of solving than the unskilled individuals (Figure 2). However, a difference in latency to solve the problem may not be a statistically robust test, as in our simulations the $95 \%$ range in the difference in latencies for small group sizes-even for the hard problems-overlaps zero (insets of Figures 2a,b).

Our simulation supports empirical findings that larger groups should exhibit overall greater innovative problem-solving performance than smaller groups (Liker and Bokony, 2009; Morand-Ferron and Quinn, 2011; Ashton et al., 2019); but it demonstrates that comparing the performance of differently-sized groups 
is generally a poor test of the pool of competence effect. Even if larger groups are more likely to contain skilled individuals, the sheer number of individuals in a group - and thus their multiplicative probabilities of solving a problem-can produce the same net positive, and non-linear relationship, between group size and different measures of group performance. Our simulations further show that irrespective of how difficult the problem is, the effect of having a skilled individual in the group is likely to be important only in small groups. Across problems with increasingly hard solution, there is a consistent inflection point (around 10 individuals) in the relationship between group size and the benefits of having a skilled individual on group performance (Figure 3).

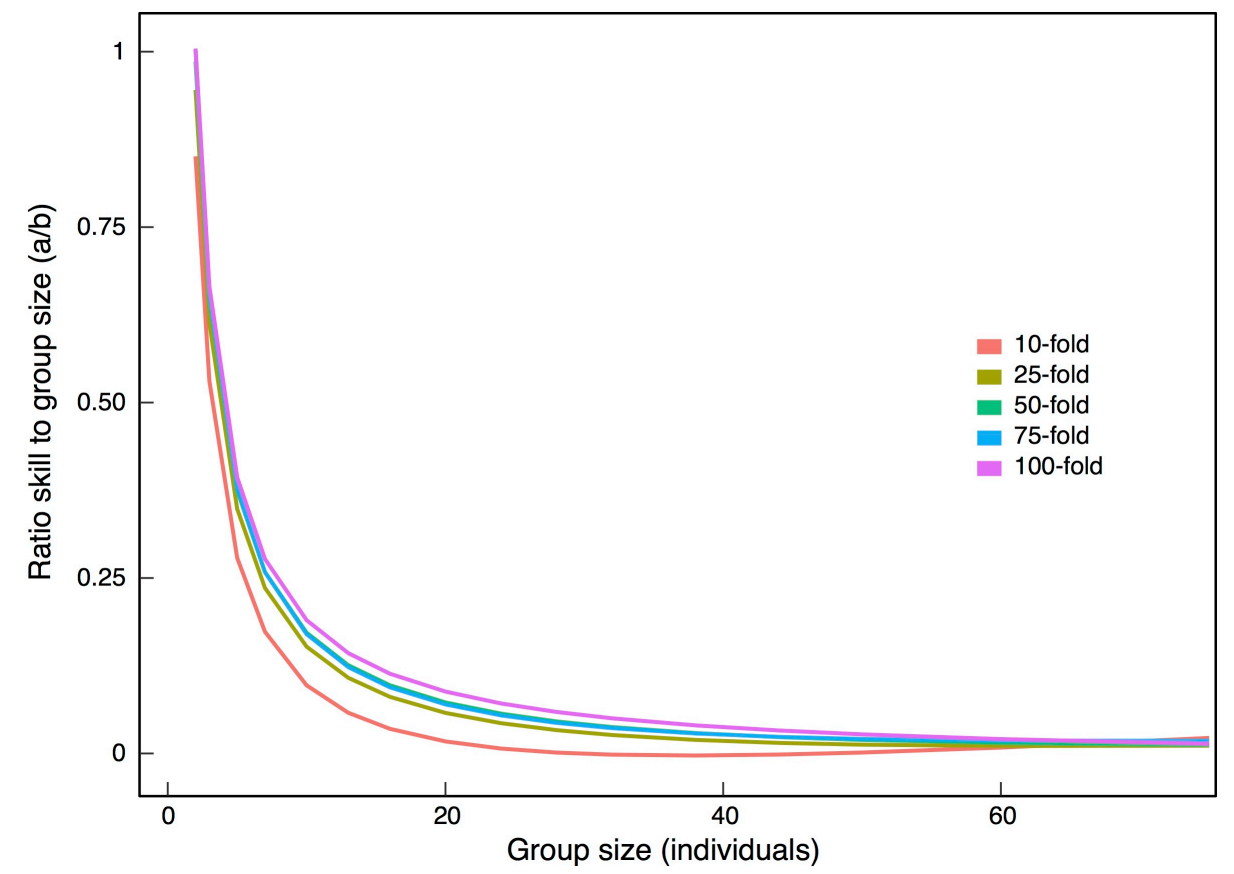

Figure 3: Larger groups perform inherently better, regardless of the difficulty of the problem to solve. The relative effects of group size versus the presence of a skilled individual in the group on the probability of solving a problem is given by the relative increase in the effect of having a skilled individual to increasing group size, given by $\frac{a_{n}}{b}$ where $a_{n}$ represents the increase in the probability of solving between groups of the same size with and without a skilled, and b represents the increase in the probability of solving in small versus large groups without a skilled individual (see Figure 1). Colour-coded lines indicate increasingly harder problems to solve. For 
instance, for an easy problem a skilled individual is 10 times more likely to solve the problem per unit effort than an unskilled individual (10-fold higher probability), whereas for a very hard problem, this probability is 100 -fold.

\section{Implications for the study of group size and overall performance}

We have shown analytically and using simulations that larger groups are expected to have a disproportionate increase in measures of group performance. Even without any differences among individuals, or any interactions among group members, we expect a non-linear relationship between group size and the time to first solving a problem and the rate at which group members subsequently learn. Further, our simulations suggest that it is likely to be impossible to distinguish a pool of competence effect from the relationship between group size and measures of group performance alone. So, if there are indeed individual differences in skills, experience and motor abilities affecting innovation (e.g. Liker and Bokony, 2009; Morand-Ferron and Quinn, 2011; Ashton et al., 2019) but the non-linear relationship between group size and innovative problem-solving is mathematically expected, how can one can detect the contributions of skilled individuals to group performance?

Our simulations suggest that the best, and perhaps only, way to study the relationship between group composition and group performance is by comparing groups of exact same size with differing compositions of skills. If specific traits can be associated with the propensity to innovate, or if the solver status of individuals can be tracked (e.g. Morand-Ferron and Quinn, 2011), then studies can also explore how group composition covaries with group size and/or 
group performance. However, four considerations should be borne in mind when designing such studies. First, when studying group composition, it must be considered that larger groups may be more likely to contain skilled individuals because these groups have, independently, been more likely to have had a group member previously innovate. Thus, while larger groups could be more likely to contain a greater pool of competence, care should be given to understanding the precise mechanisms generating such a pattern. Second, the effect of group composition is best studied at small groups, a size at which the contributions of any skilled individual would be more prominent. The group size and performance relationship between groups with and without skilled individuals is more similar, thus much harder to be distinguished, in larger than in smaller groups. Third, the differences in performance between skilled versus unskilled groups is clearer when measuring solving efficiency rather than propensity to solve (i.e. whether a group has solved in a given time). Thus, we recommend that all groups should be given as much time as required for individuals to solve a task before examining performance as a function of group composition. Fourth, the non-linear nature of the group size to group performance relationship suggests that it may not be appropriate to control for group size statistically (e.g. by fitting group size as a fixed effect), unless the relationship can be modelled accurately and explicitly.

The ability for unskilled individuals to also solve tasks makes the analysis of group composition effects challenging to analyse. One reason is that easy tasks make the performance of groups much more similar across a range of group sizes. Thus, an additional suggestion is that the effect of group composition will 
be clearer in studies where the solution to the problem to solve is not trivial, so that individual variations in skills is more easily detectable because the latencies for groups to solve are longer. One design could be to study situations where only some individuals either naturally have information about the problem or have been trained in advance. For instance, groups of tits (Paridae) known to contain an individual that had previously solved a task were faster at solving the same task in a subsequent attempt (Morand-Ferron and Quinn, 2011). Other fields, such as collective animal behaviour, have also created situations in which some individuals have information ('informed') and others do not ('uninformed individuals') to demonstrate that few knowledgeable individuals are needed to lead groups to resources (e.g. Couzin et al., 2005; Kao and Couzin, 2014). Yet if all individuals in such a test are naïve, then identifying whether some individuals have traits that enable them to be faster than others at solving problems is less likely to succeed.

While our simulation focuses on the pool of competence hypothesis, we would suggest that the general conclusions may also apply to other proposed effects, such as social facilitation, including positive correlations between group size and reduced neophobia or predator vigilance (Table 1 ). That is, such additional effects will be unlikely to change the fundamental shape of the relationship of group size and group performance, but rather mainly act to decrease the latencies to solve, both of the first innovator and of the subsequent social learners. Again, this effect is most likely to be observed at small group sizes. Similarly, while we did not consider the potential negative effects of increasing group size, including competition, scrounging and aggression, these will 
generally not alter the fundamental relationship. For example, monopolisation of the new problem by a few individuals would potentially lead to a reduction in innovation rate (see also Ashton et al., 2019), or the rate at which solving can be attempted, but this change would be unlikely to affect the latency to first solving. Moreover, the increased risk of scrounging could reduce the benefit of innovation, but while this might increase the latencies for individuals to learn, it will simply stretch the relationship, which could potentially make it appear more linear but would not actually remove the non-linear component. However, it would be an interesting question for future studies to identify cases where within-group effects, such as social obstruction, might alter the predicted expectation of the group size to group performance relationship in ways that enhance the effects of having skilled individuals in the group.

Finally, we turn to another common assumption of empirical studies on group performance: that larger groups are not only more likely to innovate, but also to promote a rapid transmission of the innovation among members, thereby increasing the performance of the average group member (Morand-Ferron and Quinn, 2011; Ashton et al., 2019). The fact that innovations spread faster and with fewer errors via social learning among socially-cohesive groups has received strong support of theoretical (Voelkl and Noe, 2008; Cantor and Whitehead, 2013) and experimental field work (Aplin et al., 2015; Whiten et al., 2016). Further, collective learning has also been proposed to convert individual experiences into superior group decisions and performance (Kao et al., 2014). However, simply observing larger groups more quickly reaching a collective solution could stem from multiple processes. While it could be the result of one 
or a few innovation events being copied and transmitted quickly within the group, it could also stem from indirect effects (e.g. larger groups having information sooner; see also Clark and Mangel, 1986) translating to a positive relationship between group size and individual learning rates. Studies should consider the contribution of different effects when evaluating hypotheses (e.g. removing the effect of larger groups having information sooner by recording latencies since the initial innovation event). In line with our general warning that the non-linear relationship between group size and group performance is not diagnostic of the pool of competence effect, the pattern of innovation spread is also not diagnostics of the type of learning (Hoppitt et al., 2010). Thus, it remains important to test for social learning of the studied innovation using two-action and control experimental paradigms, where social learning can be clearly distinguished from individual learning (Aplin et al., 2015; Whiten et al., 2016; Ashton et al., 2019).

\section{Final remarks}

Larger groups of animals innovate disproportionally more often, and faster, than small groups. Empirical studies have been influential in advancing explanations for the relationship between group size and group performance. However, we emphasise the need to further consider a fundamental mechanistic explanation for why larger groups so convincingly outperform smaller groups. Our model highlights two important points. First, we show that the non-linear relationship between probability of solving a problem is expected to arise due to mathematical properties alone, i.e. from the strength in numbers, without 
needing to invoke any further explanatory factors. Second, it is not possible to draw conclusions for or against any hypothesis by solely looking at the shape of the curve representing the relationship between group size and performance. A positive non-linear relationship should not be mistaken as evidence for any effect of group size on group performance beyond group size alone.

In clarifying the fundamentals of the group size and performance relationship, we do not intend to criticise existing research, but inspire more mechanistic investigations into the benefits that can arise from living in a larger group and, most importantly, the development of more robust null hypotheses against which to evaluate empirical data. Studies interested in inferring group composition in terms of skill levels should control for group size in their data collection by comparing the performance of groups of identical sizes, rather than in their statistical tests. Doing so will help us better tease apart the roles of individual variation and learning in the innovative problem-solving skills of animal collectives as they cope with an increasingly changing world.

\section{ACKNOWLEDGEMENTS}

This work was funded by the Max Planck Society, by the DFG Centre of Excellence 2117 "Centre for the Advanced Study of Collective Behaviour" (422037984), by a DFG grant (FA 1420/4-1), and by a grant from the European Research Council (ERC) under the European Union's Horizon 2020 research and innovation programme (grant agreement No. 850859) awarded to DRF. LMA was funded by a Max Planck Society Independent Research Group Leader Fellowship. MC was funded by a Coordenação de Aperfeiçoamento de Pessoal de 
Nível Superior (CAPES-Brazil) postdoctoral fellowship (88881.170254/201801).

\section{REFERENCES}

Amici, F., Widdig, A., Lehmann, J., Majolo, B., 2019. A meta-analysis of interindividual differences in innovation. Animal Behaviour 155, 257-268.

Aplin, L.M., Farine, D.R., Morand-Ferron, J., Cockburn, A., Thornton, A., Sheldon, B.C., 2015. Experimentally induced innovations lead to persistent culture via conformity in wild birds. Nature 518, 538-541.

Ashton, B.J., Ridley, A.R., Edwards, E.K., Thornton, A., 2018. Cognitive performance is linked to group size and affects fitness in Australian magpies. Nature 554, 364-367.

Ashton, B.J., Thornton, A., Ridley, A.R.J.A.B., 2019. Larger group sizes facilitate the emergence and spread of innovations in a group-living bird. Animal Behaviour $158,1-7$.

Berdahl, A., Torney, C.J., Ioannou, C.C., Faria, J.J., Couzin, I.D., 2013. Emergent sensing of complex environments by mobile animal groups. Science 339, 574576.

Biondi, L.M., Bo, M.S., Vassallo, A.I., 2010. Inter-individual and age differences in exploration, neophobia and problem-solving ability in a Neotropical raptor (Milvago chimango). Animal Cognition 13, 701-710.

Bolnick, D.I., Svanback, R., Fordyce, J.A., Yang, L.H., Davis, J.M., Hulsey, C.D., Forister, M.L., 2003. The ecology of individuals: Incidence and implications of individual specialization. The American Naturalist 161, 1-28. 
Burns, J.G., Dyer, A.G., 2008. Diversity of speed-accuracy strategies benefits social insects. Current Biology 18, R953-R954.

Cantor, M., Whitehead, H., 2013. The interplay between social networks and culture: theoretically and among whales and dolphins. Philosophical Transactions of the Royal Society B 368.

Caraco, T.J.E., 1981. Risk - sensitivity and foraging groups. Ecology 62, 527-531.

Clark, C.W., Mangel, M.J.T., 1986. The evolutionary advantages of group foraging. Theoretical Population Biology 30, 45-75.

Coleman, S.L., Mellgren, R.L., 1994. Neophobia When Feeding Alone or in Flocks in Zebra Finches, Taeniopygia-Guttata. Animal Behaviour 48, 903-907.

Couzin, I.D., Krause, J., Franks, N.R., Levin, S.A., 2005. Effective leadership and decision-making in animal groups on the move. Nature 433, 513-516.

Ducatez, S., Sol, D., Sayol, F., Lefebvre, L., 2020. Behavioural plasticity is associated with reduced extinction risk in birds. Nature Ecology \& Evolution 16.

Freeberg, T.M., Eppert, S.K., Sieving, K.E., Lucas, J.R.J.S.r., 2017. Diversity in mixed species groups improves success in a novel feeder test in a wild songbird community. Scientific Reports 7, 43014.

Gibelli, J., Aubin-Horth, N., Dubois, F., 2019. Individual differences in anxiety are related to differences in learning performance and cognitive style. Animal Behaviour 157, 121-128.

Giraldeau, L.A., 1984. Group foraging - the Skill Pool Effect and frequencydependent learning. The American Naturalist 124, 72-79.

Griffin, A.S., Guez, D., 2014. Innovation and problem solving: A review of common mechanisms. Behavioural Processes 109, 121-134. 
Hirsch, B. T., 2007. Costs and benefits of within-group spatial position: a feeding competition model. The Quarterly Review of Biology 82, 9-27.

Hong, L., Page, S.E., 2004. Groups of diverse problem solvers can outperform groups of high-ability problem solvers. Proceedings of the National Academy of Sciences of the USA 101, 16385-16389.

Hoppitt, W., Kandler, A., Kendal, J.R., Laland, K.N., 2010. The effect of task structure on diffusion dynamics: Implications for diffusion curve and network-based analyses. Learning and Behaviour 38, 243-251.

Kao, A.B., Couzin, I.D., 2014. Decision accuracy in complex environments is often maximized by small group sizes. Proceedings of the Royal Society of London BBiological Sciences 281, 1784.

Kao, A.B., Miller, N., Torney, C., Hartnett, A., Couzin, I.D., 2014. Collective learning and optimal consensus decisions in social animal groups. Plos Computational Biology 10.

Kerr, N.L., Tindale, R.S., 2004. Group performance and decision making. Annual Reviews of Psychology 55, 623-655.

Keynan, O., Ridley, A.R., Lotem, A., 2015. Social foraging strategies and acquisition of novel foraging skills in cooperatively breeding Arabian babblers. Behavioral Ecology 26, 207-214.

Krause, J., Ruxton, G.D., Krause, S., 2010. Swarm intelligence in animals and humans. Trends in Ecology and Evolution 25, 28-34.

Liker, A., Bokony, V., 2009. Larger groups are more successful in innovative problem solving in house sparrows. Proceedings of the National Academy of Sciences of the USA 106, 7893-7898. 
Melis, A.P., Hare, B., Tomasello, M., 2006. Chimpanzees recruit the best collaborators. Science 311, 1297-1300.

Morand-Ferron, J., Quinn, J.L., 2011. Larger groups of passerines are more efficient problem solvers in the wild. Proceedings of the National Academy of Sciences of the USA 108, 15898-15903.

Pitcher, T.J., Magurran, A.E., Winfield, I.J., 1982. Fish in larger shoals find food faster. Behavioral Ecology and Sociobiology 10, 149-151.

Ranta, E., Rita, H., Lindstrom, K.J.T.A.N., 1993. Competition versus cooperation: success of individuals foraging alone and in groups. The American Naturalist $142,42-58$.

Reader, S. M., Laland, K. N., 2003. Animal innovation. Oxford: Oxford University Press.

Reader, S. M., Laland, K. N., 2002. Social intelligence, innovation, and enhanced brain size in primates. Proceedings of the National Academy of Sciences 99, 4436-4441.

Ryer, C.H., Olla, B.L., 1992. Social mechanisms facilitating exploitation of spatially-variable ephemeral food patches in a pelagic marine fish. Animal Behaviour 44, 69-74.

Sih, A., Sinn, D.L., Patricelli, G.L., 2019. On the importance of individual differences in behavioural skill. Animal Behaviour 155, 307-317.

Smit, J.A.H., van Oers, K., 2019. Personality types vary in their personal and social information use. Animal Behaviour 151, 185-193.

Sol, D., Duncan, R. P., Blackburn, T. M., Cassey, P., Lefebvre, L., 2005. Big brains, enhanced cognition, and response of birds to novel environments. Proceedings of the National Academy of Sciences 102, 5460-5465. 
Sol, D., Timmermans, S., Lefebvre, L., 2002. Behavioural flexibility and invasion success in birds. Animal Behaviour 63 495-502.

Soma, M., Hasegawa, T., 2004. The effect of social facilitation and social dominance on foraging success of budgerigars in an unfamiliar environment. Behaviour 141, 1121-1134.

Stöwe, M., Bugnyar, T., Heinrich, B., Kotrschal, K., 2006. Effects of group size on approach to novel objects in ravens (Corvus corax). Ethology 112, 1079-1088.

Thornton, A., Samson, J., 2012. Innovative problem solving in wild meerkats. Animal Behaviour 83, 1459-1468.

Thornton, A., Malapert, A., 2009 Experimental evidence for social transmission of food acquisition techniques in wild meerkats. Animal Behaviour 78, 255-264.

Voelkl, B., Noe, R., 2008. The influence of social structure on the propagation of social information in artificial primate groups: A graph-based simulation approach. Journal of Theoretical Biology 252, 77-86.

Whiten, A., Caldwell, C.A., Mesoudi, A., 2016. Cultural diffusion in humans and other animals. Current Opinion in Psychology 8, 15-21. 